\title{
Pleomorphic Hyalinizing Angiectatic Tumor of Soft Parts
}

National Cancer Institute

\section{Source}

National Cancer Institute. Pleomorphic Hyalinizing Angiectatic Tumor of Soft Parts. NCI

Thesaurus. Code C53594.

A non-metastasizing soft tissue neoplasm characterized by the presence of a neoplastic stroma containing spindle cells, dilated thin-walled vessels lined by fibrin, and a chronic inflammatory infiltrate. The majority of cases develop in the subcutaneous tissues usually in the lower extremities and present as slow growing masses. Recurrences occur in approximately half of the cases. 